\title{
Description of the buckling of a chain of hard spheres in terms of Jacobi functions
}

\author{
D. Weaire ${ }^{\text {a }}$, A. Mughal ${ }^{\text {b,* }}$, J. Ryan-Purcell ${ }^{\text {a }}$, S. Hutzler ${ }^{\text {a }}$ \\ a School of Physics, Trinity College Dublin, Dublin 2, Ireland \\ ${ }^{\mathrm{b}}$ Department of Mathematics, Aberystwyth University, Penglais, Aberystwyth, Ceredigion, Wales SY23, United Kingdom
}

\section{A R T I C L E I N F O}

\section{Article history:}

Received 17 June 2021

Received in revised form 10 January 2022

Accepted 21 January 2022

Available online 2 February 2022

Communicated by Tamas Insperger

\section{Keywords:}

Buckling

Hard sphere chain

Duffing equation

Jacobi functions

\begin{abstract}
A B S T R A C T
A linear chain of hard spheres, confined by a transverse harmonic potential, buckles under compression between two hard walls. Jacobi functions provide exact analytic solutions of a differential equation (related to the Duffing equation) for the displacement profile of this chain, within a continuum approximation. Here we explore these solutions, describing their forms and the way in which they vary as system parameters are changed. This is illustrated by reference to two-dimensional diagrams in which each such solution is represented by a point and contour plots illustrate various characteristics of the solutions (period, compression, localization, etc.). Our findings enrich the study of the buckling instability for a linear chain of particles. The approach presented here has the advantage of being based on a simple chain of hard spheres and is straightforward in its interpretation. As such these results may provide insight into more complex experiments, such as those involving the buckling of ion chains.
\end{abstract}

(c) 2022 Elsevier B.V. All rights reserved.

\section{Introduction}

When a confined linear chain of hard spheres (e.g. ball bearings in a horizontally placed cylinder) is compressed between hard walls, the chain buckles. The result is a modulated zig-zag structure of lateral sphere displacements, with a displacement profile which becomes increasingly localized as compression is increased.

This interesting nonlinear system is readily accessible to experiment (including some very simple procedures [1-4]) and may be simulated by straightforward numerical methods [2,4]. Its properties include the appearance of further equilibrium states of sphere displacements at high values of compression and the eventual occurrence of additional contacts between spheres, at which point new structures begin to form [4].

Systems in which analogous examples of one-dimensional pattern formation have been observed include cold ions in traps [5-13] (with possible relevance to quantum computing [14,15]), dusty plasmas [16], droplets in microfluidic crystals [17], paramagnetic colloidal particles in an external field [18], and linear chains of magnetic spheres [19]. In contrast, the system that we study here, consisting of hard spheres in a harmonic confining potential, is much more tractable in both theory and experiment $[3,20]$, yet displays the rich buckling phenomena observed in the more complex systems listed above.

\footnotetext{
* Corresponding author.

E-mail address: aqm@aber.ac.uk (A. Mughal).
}

In the experimental system which stimulated the work presented here, a small number of ball-bearings (between five and eight) was placed inside a horizontal cylindrical tube, sealed with stoppers at both ends [4]. One of the stoppers is movable, allowing for a compression of the chain of spheres. For a tube diameter much greater than that of the spheres the resulting buckling structures are approximately planar. The transverse harmonic potential that we use in our model is provided by gravity, as spheres are displaced transversely in the tube upon compression. While we cannot eliminate friction in our existing set-up [4] we have reduced it by immersing the spheres in vegetable oil.

When such a chain of hard spheres is compressed, it buckles to form a modulated zig-zag structure, of which several examples can be seen in [3,4], and in Section 2.

In previous work [2-4] we have developed a numerical simulation of this system; a summary of that methodology is presented in Appendix A. In these discrete simulations it was evident that there is a wide range of the total number of spheres $N$ and values of compression for which the profile of the magnitude of transverse displacement as a function of sphere number is very smooth. As localization increases (with increasing compression), this is less true, since the localization peak, within which the amplitude of the displacement is strongly varying, includes only a few spheres.

We have developed a continuum theory, expected to conform well to the discrete simulations for large $N$ and small compression. In this theory, which was first introduced in [4], discrete recursion relations are replaced by differential equations. This 

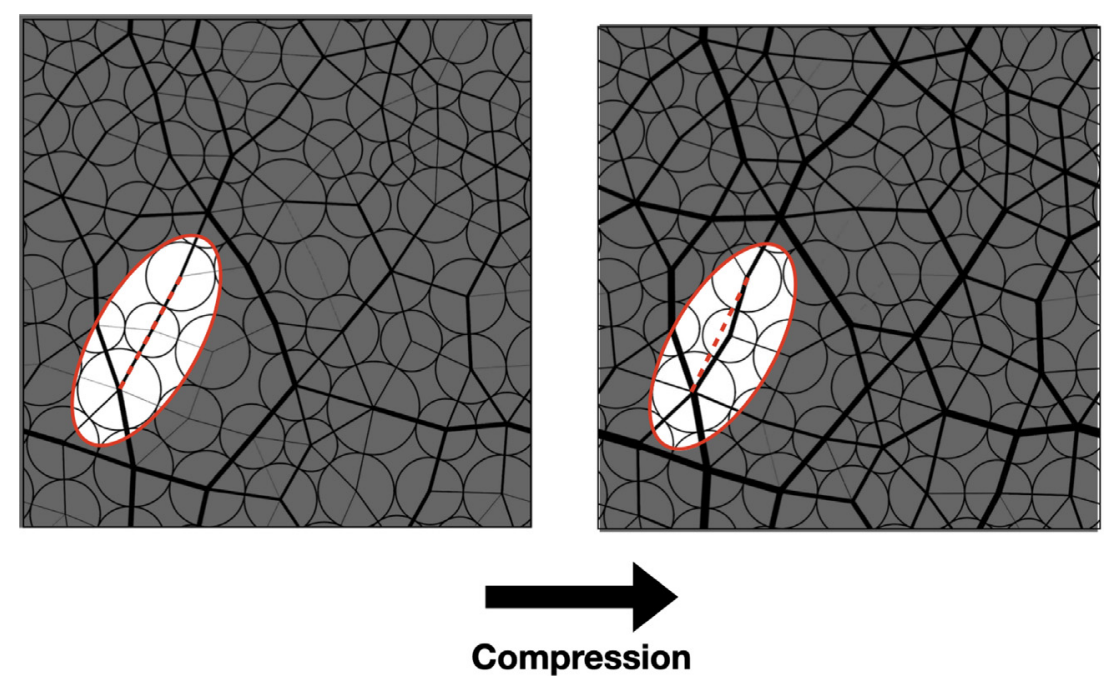

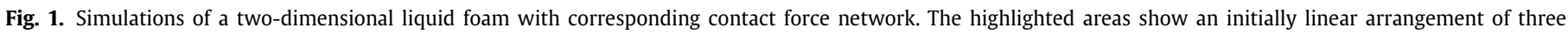

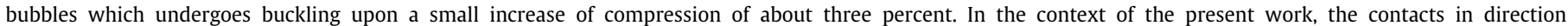
perpendicular to the axis of the three-bubble chain provide a transverse confining potential for the chain. (For simulation details of the bubble packing see [22].).

procedure is analogous to the development of the Sine-Gordon equation in the theory of solitons [21].

In both discrete and continuous descriptions, there are equations that must be satisfied for equilibrium, and their general solutions are periodic. In the case of the hard wall boundary conditions used here, the simplest equilibrium state is such that one half period of such a solution is required. This state is necessarily stable with respect to small perturbations. Further stable and unstable states exist: for example represented by multiples of the half-period of $N$. These will not be considered here, and this restriction is implied throughout.

We have structured this article as follows. We first describe the discrete model, for completeness. We then focus on the continuum description, deriving a differential equation for the equilibrium profile, an improvement on our previous version [4]. While this equation can be solved numerically, we instead resort to a further approximation that is analytically tractable. This simpler equation is an example of the well known Duffing equation (without damping and forcing terms). The Jacobian elliptic functions play a major role here as solutions to the Duffing equation: we discuss the relevant functions in detail. Quantities directly relevant to experiments, such as compression and localization, may be easily evaluated and related to each other in this way. We use diagrams to make manifest these results and provide a guide to the relationships of the many parameters in this system. Much of the insight thus gained would be very difficult to acquire directly from the original exact but discrete description of the system.

Instabilities that lead to buckling are prominent in the theory of engineering structures and in materials science [23,24]. In the present case buckling takes place under infinitesimal compression, whereas finite compression is required for buckling of a chain of elastic spheres. In both cases the buckling is in the form of a smoothly modulated zig-zag displacement: its localization for higher compression is central to the present paper. This is analogous to the localized deformation that occurs when some materials are compressed [25], depending critically on their elastic/plastic properties. Further examples of systems with similar properties include: [26], which explores the buckling of a growing linear filament confined to a substrate, the reversible buckling of an elastic shell (with potential for information storage) [27], as well as a vast literature devoted to the buckling of thin-walled cylinders under loading (see [28] for a recent example).
Our work is also related to models used in studies of "force chains" in 2D or 3D granular materials [29,30], a wide class of materials that includes colloidal packings [31] and wet foams [22]. When these have a disordered structure, the larger contact forces form a random network of more or less straight chains, playing the major role in supporting an imposed stress. Each of these is rather similar to our single chain: the confining transverse potential in our case corresponds to the weaker forces due to surrounding grains or bubbles. An example of the sudden local buckling of a chain, induced by increasing compression, is shown in Fig. 1 for a two-dimensional packing of bubbles [22], as simulated by the Morse-Witten method [32,33]. Note that buckling takes place over a small range of compression. However, in this case, such recognizable events are mainly associated with very short chains, typically only involving three bubbles.

This appealing scenario, which suggests that local buckling plays a role in response to applied stress in such materials, has been studied in particular by Hunt et al. [34]. The main difference to the work presented here is the incorporation of bending forces between spheres in Hunt's model. This makes the detailed buckling behaviour rather different, as the presence of these bending forces discourages the modulated zig-zag displacement of spheres that we find in our model. However, qualitative similarities may be seen in the examples shown by these authors and ourselves.

\section{The discrete chain}

\subsection{The buckling profile}

Fig. 2(a) shows a buckling profile which develops when a linear chain of hard spheres is compressed by a small amount; the dimensionless compression $\Delta$ is defined as the reduction of length from that of the straight linear (touching) chain, divided by the sphere diameter, corresponding to

$\Delta=N-\sum_{n=0}^{N} \cos \theta_{n}$.

The buckling profile takes the form of a modulated zig-zag structure, with alternating transverse displacements of (here $N=$ 10) successive spheres. Fig. 2(b) shows the corresponding profile of angles $\theta_{n}$ (with $0 \leq n \leq N$, see also Fig. A.1 in Appendix A), note the symmetry about the centre. 


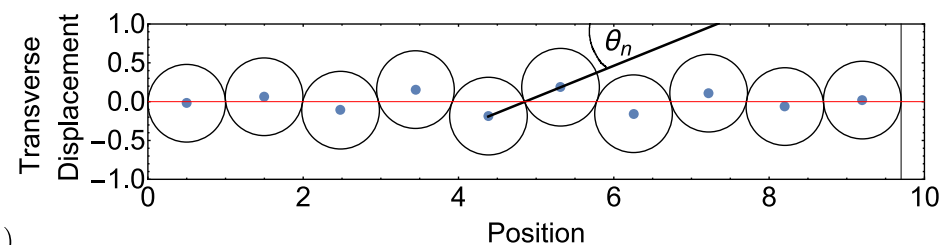

(a)

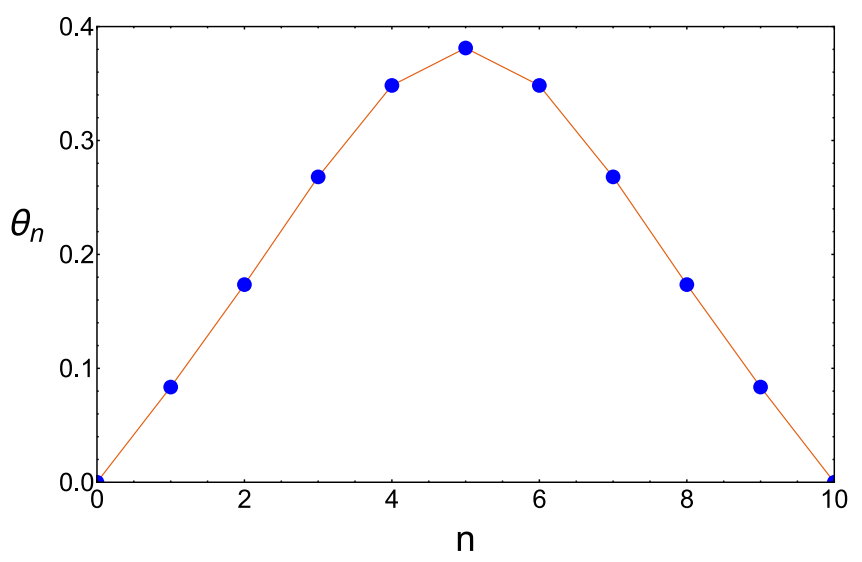

(b)

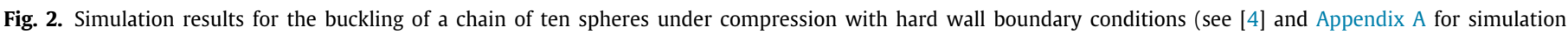

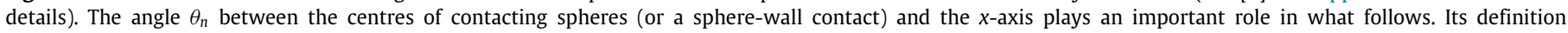

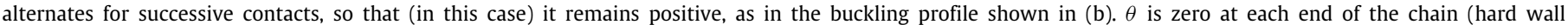

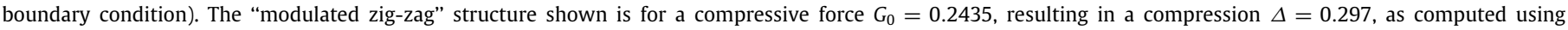
Eq. (1).

The definition of $\theta_{n}$ that we use is such that it is always of the same sign for the states considered in this paper, and chosen to be positive (the states with negative sign have identical form and properties) for a modulated zig-zag structure, to which all the structures described in this paper conform.

\subsection{Compressive force}

The compressive force $G_{0}$ exerted at each hard wall plays an important role in what follows. The straight chain remains stable for $G_{0}$ less than a critical value that depends on the number of spheres $N$. By considering the balance of forces on a single displaced sphere one may deduce an upper bound $G_{0}=1 / 2$ for the critical force (expressed in the dimensionless form introduced in Appendix A). The value $G_{0}=1 / 4$ is more significant as the buckled structures take different qualitative forms above and below this threshold (see also Appendix B for the case of the uniform zig-zag structure).

\section{The continuum formulation}

\subsection{Continuum equations}

The buckling profile, of which an example was shown in Fig. 2, has a shape which evolves as compression is increased, with the central peak becoming narrower, so that buckling is more localized. In seeking to describe this we have found that a reformulation in terms of a continuous variable $\theta(u)$ (the angle indicated in Fig. 2), where $u$ is the continuous counterpart of the integer variable $n$, offers advantages [4], and it is the main basis of what follows. The transition from the discrete to the continuous description is described in detail in Appendix C.

The resulting differential equation for $\phi(u)=\tan \theta(u)$ is as follows,

$\phi^{\prime \prime}(u)=-4 \phi(u)+\left(4+\kappa^{2}\right) \frac{\phi(u)}{\sqrt{1+\phi^{2}(u)}}$, where we represent the compressive force $G_{0}$ exerted at both ends of the chain by the quantity $\kappa^{2}$, which is defined as

$\kappa^{2}=G_{0}^{-1}-4$.

It may be positive or negative, so that the square root $\kappa$ may be real or imaginary. However, a finite positive compressive force $G_{0}$ requires $\kappa^{2}>-4$. (In our original paper [2] the quantity $\kappa^{2}$ was denoted by $\epsilon$.)

The differential equation stated in [4] was of a different form than that of Eq. (2) (arising from a different method for the approximation of derivatives). Although resulting in very similar numerical solutions for $\theta(u)$, it did not preserve the symmetry of the solution; the derivation in Appendix $C$ satisfies that requirement, as well as using $\phi=\tan \theta$ as the dependent variable.

Fig. 3 shows a numerical solution of Eq. (2) corresponding to the calculation for the discrete case in Fig. 2, for the hard wall boundary conditions $(\theta=\phi=0$ at the two end points of the chain, at $u=0$ and $u=N$ ). Compression $\Delta$ (Eq. (1)) in the continuum formulation is given by the integral

$\Delta=\int_{0}^{N}(1-\cos \theta(u)) d u=\int_{0}^{N} \frac{\sqrt{1+\phi^{2}(u)}-1}{\sqrt{1+\phi^{2}(u)}} d u$

where the integration has generally to be performed numerically for given $\phi(u)=\tan \theta(u)$.

By expanding the square root in Eq. (2) to order $\phi^{2}$ the equation may be reduced to the following,

$\phi^{\prime \prime}=\kappa^{2} \phi-2\left(1+\frac{\kappa^{2}}{4}\right) \phi^{3}$,

which we may call the reduced equation. It is correct to order $\mathcal{O}\left(\phi^{3}\right)$ (see also Appendix C).

In Section 6 we show that its analytical solutions compare well with numerical solutions of the full equation, Eq. (2), for sufficiently small values of compression. (This is also the case for solutions of $\theta^{\prime \prime}=\kappa^{2} \theta-2 \theta^{3}$, the reduced equation that we had 


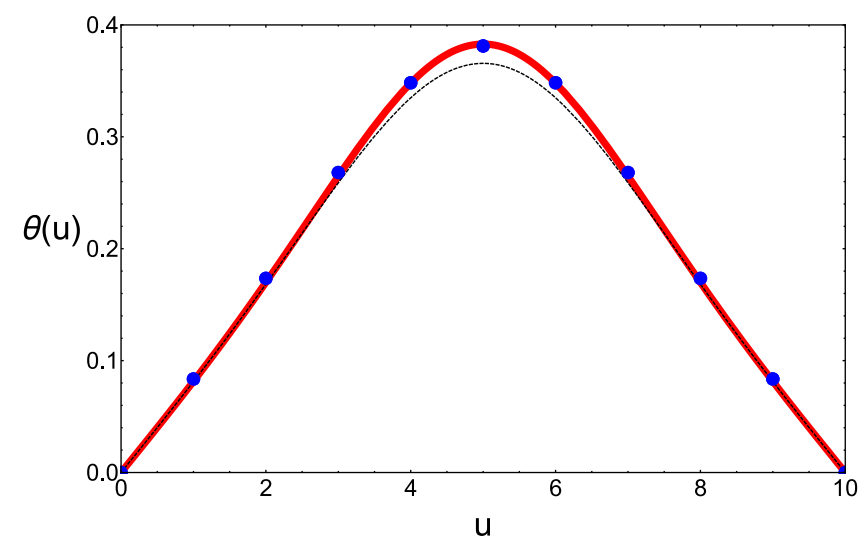

Fig. 3. Results for the continuum model applied to the example shown in Fig. 2, i.e. for ten spheres with a compressive force $G_{0}=0.2435$ (corresponding to $\kappa^{2}=0.1068$, Eq. (3)). The solid line is a numerical solution for $\theta(u)$ for the full continuum differential equation Eq. (2) under these conditions. The dashed line is the analytical solution of the reduced equation, Eq. (5). Also shown are the data points for the corresponding discrete system, as in Fig. 2(b).

originally formulated [4].) Fig. 3 shows solutions of both full and reduced equation for the example of $\kappa^{2}=0.1068$.

The reduced equation, Eq. (5), is one particular case of the Duffing equation [35], well known in the theory of oscillations, where it serves as an analytically tractable approximation to a wide range of dynamical systems, including the nonlinear pendulum, the forced oscillations of beams and cables, as well as nonlinear electrical circuits [35]. In its most general form the Duffing equation is given by

$\ddot{x}(t)=-\delta \dot{x}-\beta x-\alpha x^{3}+\gamma \cos (\omega t)$.

In the absence of both a damping term $(\delta=0)$ and a driving force $(\gamma=0)$ this is equivalent to Eq. (5).

The character of the phenomena described by the Duffing equation depends on the nonlinear term $-\beta x-\alpha x^{3}$. For $\beta>0$ it describes an oscillator with a nonlinear restoring force, while for $\beta<0$ it describes the dynamics of a point mass in a double well potential. In our case this cross-over is governed by the value of $\kappa^{2}$, as we shall show in the following section.

While much of the analysis of the Duffing equation has been directed towards understanding dynamic properties (e.g. the pendulum), the present problem represents a realization of a particular case of the equation in a static system.

\subsection{Properties of the reduced equation}

Many of the general properties of the full equation, Eq. (2), and its reduced form, Eq. (5), are obvious and well illustrated by their phase portraits shown in Fig. 4. These include symmetry under $\theta \rightarrow-\theta$ and $u \rightarrow-u$, periodicity (with an exception noted below) and the dependence on the sign of $\kappa^{2}$. The key feature that can be seen in the phase portraits as $\kappa^{2}$ goes from $\kappa^{2}<0$ to $\kappa^{2}>0$ is the transition from a system with one stationary point to a system with two stationary points (the case of $\kappa^{2}=0$ is intermediate between these two regimes).

For $\kappa^{2}>0$ the solution of the reduced equation, Eq. (5), has inflection points $\left(\phi^{\prime \prime}=0\right)$ wherever

$\phi=0$, or $\pm \sqrt{\frac{\kappa^{2}}{2\left(1+\kappa^{2} / 4\right)}}$.

(In this paper $\theta$ (and thus $\phi=\tan \theta$ ) is always positive, with $\phi \ll 1$.) For the allowed range of negative values of $\kappa^{2}(-4<$ $\kappa^{2}<0$, corresponding to a positive compressive force $G_{0}$, Eq. (3)) the second type of inflection point does not arise.

For the hard wall boundary conditions applied here $(\phi(0)=$ $\phi(N)=0$ ), the solutions are symmetric about the midpoint of the system. It is clear therefore that for $\kappa^{2}<0$, the relevant solution is "cosine-like" (see Section 5, Fig. 6, example G).

For $\kappa^{2}>0$ the solution develops a more or less prominent peak at the centre (localization of buckling), see Fig. 6, example C). This type of solution may extend to infinity with exponential tails, $\theta \sim \exp ( \pm \kappa u)$, if the separation of the hard walls is taken to the infinite limit.

The intermediate case $\kappa=0$ has solutions with no second, third, and fourth derivatives at the two edges, so that it takes a roughly triangular form (see Fig. 6, example E). In this case the following approximate relationship holds for compression $\Delta$ (Eq. (4)), $\Delta \approx \frac{4}{3} N^{-1}$.

\section{Jacobi functions}

While not entirely elementary, the reduced equation, Eq. (5), does have analytic solutions in the form of Jacobi functions, as has been recognized in other contexts in which the Duffing equation is applied. The solutions which are categorized in the following are for the boundary condition $\theta=0$ (and thus also $\phi=0$ ) at $u=$ $-N / 2$ and $u=N / 2$ (corresponding to a centering of the profiles shown in Fig. 2 and Fig. 3 on $u=0$ ). This requires a Jacobi function with real zeros, from which we extract the part between two zeros.

To our knowledge, within the realm of physics these "mythical functions" [36] occur mainly in the theory of various problems in classical dynamics and electronic/optical oscillators [36-38], with only a limited number of applications in statics, e.g. for the bending of thin rods [39]. The mathematical background features "notations and conventions that are often contradictory or difficult to understand by physicists" [36]. Hence we will spell out our findings with explicit detail. We will formulate the analytical theory and in Section 5 encapsulate our findings in a number of diagrams which should make the role of Jacobi functions transparent. Before doing so, we will introduce relevant notation and then define relevant solutions of the reduced equation (for hard wall boundary conditions) in terms of scaled Jacobi functions (see also Appendix D).

The reduced equation, Eq. (5), is only an approximation to the full one, but offers many opportunities to develop the mathematics of its solutions, including bringing the Jacobi functions into play. Jacobi functions play a similar role in the theory of the pendulum, where they arise in two ways. In the first case the Jacobi functions can be used to provide exact solutions for the sine of the angle of displacement $[38,40,41]$. In the second case, an approximate solution to the differential equation for the pendulum can be obtained by truncating to the third order in the angle of displacement; this leads to a reduced equation that has solutions directly in terms of a Jacobi function (see Appendix E and $[35,41])$.

\subsection{Notation for Jacobi functions}

There are twelve different Jacobian elliptic functions whose primary definition is framed in terms of the inversion of integrals $[42,43]$. These functions are solutions of first and second order differential equations [42] and it will be this aspect which concerns us here.

Only four Jacobi functions satisfy differential equations which may be transformed into the reduced equation, Eq. (5). These four functions may be rescaled, as detailed below, to give explicit analytic solutions of the equation. However, our present restriction 
(a)
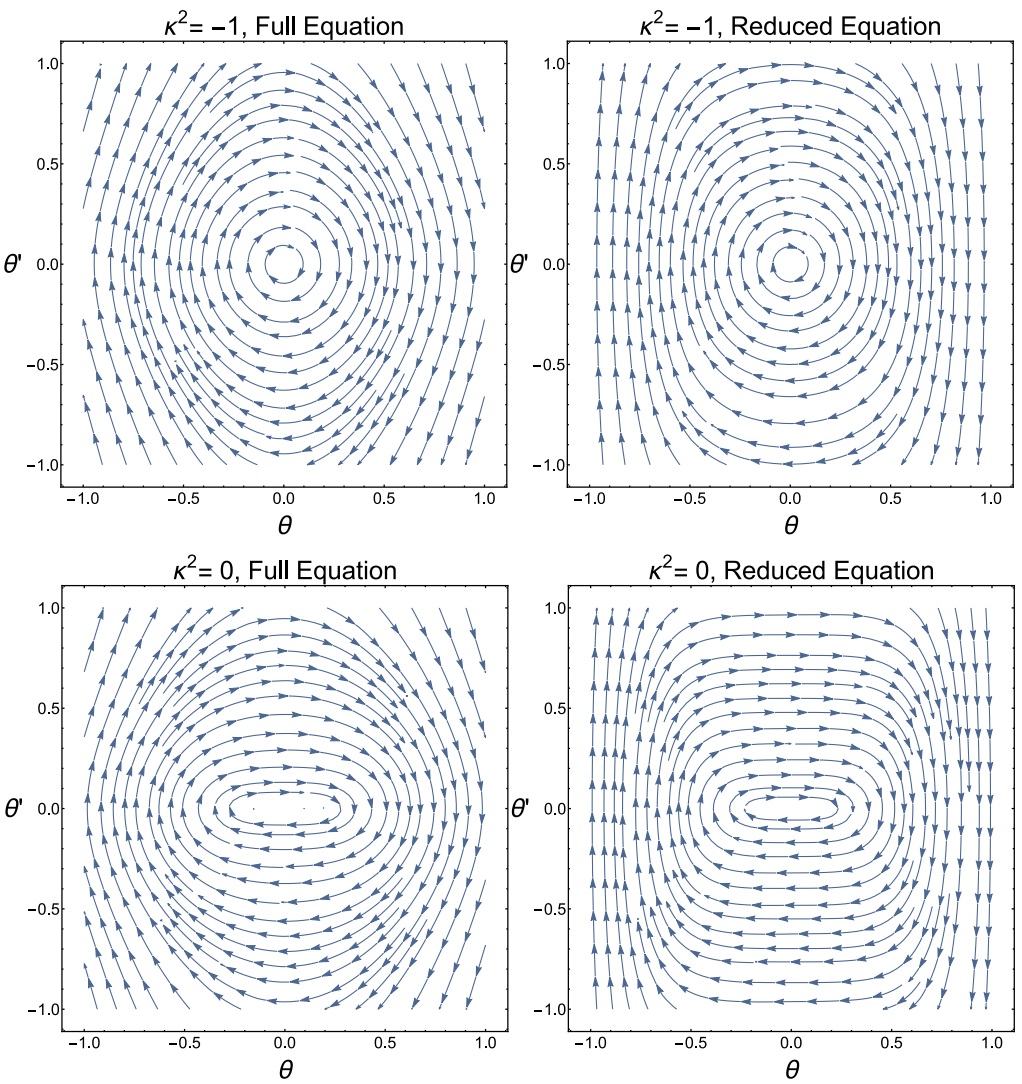

(b)
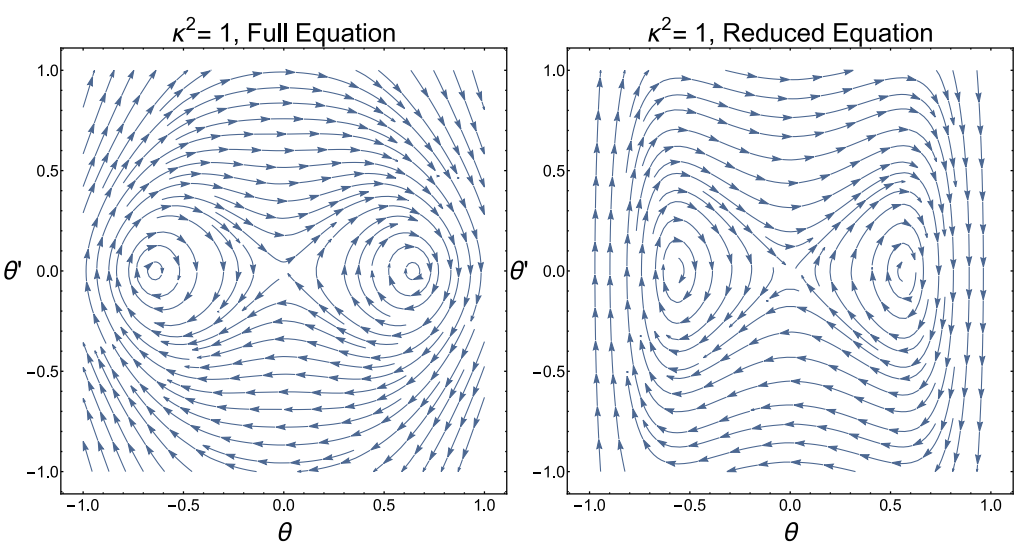

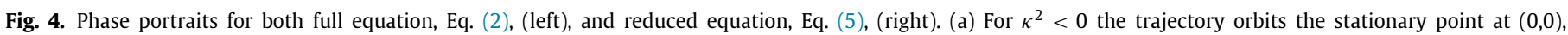

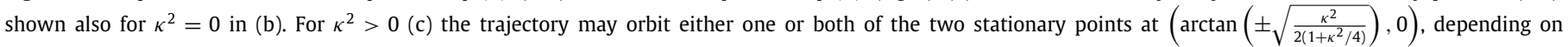
the boundary conditions.

to hard wall $(\theta=0)$ boundary conditions further reduces the available function types to two, which have zeros. These are $\mathrm{cn}$ and $s d$, but these are related by a translation (like the $\sin$ and $c o s$ functions), so that only one of them is required: we choose $\mathrm{cn}$.

There is a one-parameter set of $c n$ functions, each being generally denoted by $c n(x \mid m)$, and the individual function is identified by $m$, where $\sqrt{m}$ is the so-called modulus. It is real for our purposes. The parameter $m$ is related to the period of the Jacobi functions, which is stated in terms of the complete elliptic integral of the first kind; see Section 4.2.

By convention, $m$ is restricted to the range $0<m<1$. Elliptic functions with a value of $m$ outside this range can be rewritten as other elliptic functions where $m$ lies between 0 and 1 [42,43]. Hence the set of functions used here is that of $c n(x, m)$ for this range.

\subsection{Jacobi function solution of the reduced equation}

The Jacobi $c n(x \mid m)$ function is a periodic even function with zeros, and a maximum of 1 at $x=0$, see Fig. 5. Its (real) period is $4 K(\mathrm{~m})$, where $K(\mathrm{~m})$ is the complete elliptic integral of the first kind.

For the range $0<m<1$ the function $y(x)=c n(x \mid m)$ satisfies the differential equation $[36,42]$

$y^{\prime \prime}=-(1-2 m) y-2 m y^{3}$.

which is to be compared with the reduced equation, Eq. (5). Matching the two forms, Eq. (8) and the reduced equation, it is easily shown (see Appendix D) that a solution of Eq. (5) for given $\kappa^{2}$ (with $\kappa^{2}>-4$ for finite positive compressive force $G_{0}$ (Eq. (3))) and satisfying the hard wall boundary conditions may 
Table 1

Solutions of the reduced equation for three special cases (see also Appendix D).

\begin{tabular}{lll}
\hline$m$ & $\phi(u)$ & Half period (number of spheres) $N$ \\
\hline $1\left(\right.$ for $\left.\kappa^{2}>0\right)$ & $\sqrt{\kappa^{2} /\left(1+\kappa^{2} / 4\right)} \operatorname{sech}\left(\sqrt{\kappa^{2}} u\right)$ & $\infty$ \\
$\frac{1}{2}\left(\right.$ for $\left.\kappa^{2}=0\right)$ & $c n\left(\sqrt{2} u \mid \frac{1}{2}\right)$ & $2 K\left[\frac{1}{2}\right] \simeq 3.70815$ \\
$\rightarrow 0\left(\right.$ for $\left.\kappa^{2}<0\right)$ & $\rightarrow m \sqrt{-\kappa^{2} /\left(1+\kappa^{2} / 4\right)} \cos \left(-\sqrt{\kappa^{2}} u\right)$ & $\pi / \sqrt{-\kappa^{2}}$ \\
\hline
\end{tabular}

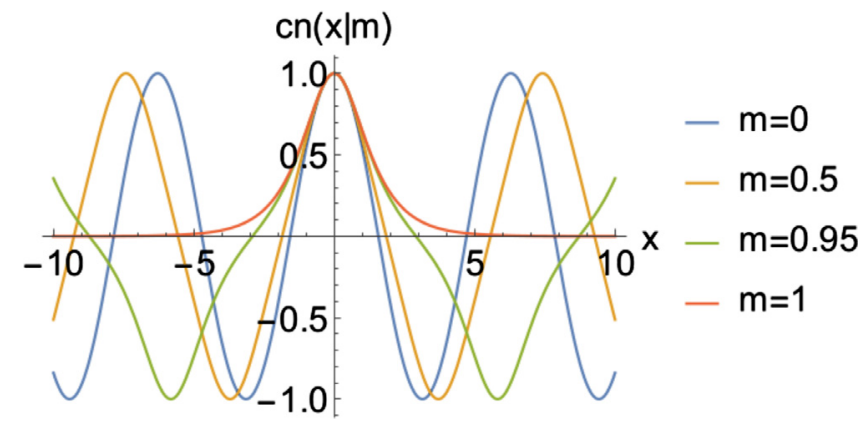

Fig. 5. Examples of the Jacobi function $\operatorname{cn}(x, m)$ for different values of the modulus $m^{1 / 2}$. Their shape varies smoothly between $\cos (x)$ (for $m=0$ ) and the hyperbolic secant, $\operatorname{sech}(x)=\frac{2}{e^{x}+e^{-x}}($ for $m=1)$.

be written as a scaled Jacobi $c n$ function,

$\phi(u)=\tan \theta(u)=\phi_{\max } \operatorname{cn}\left(\sqrt{\kappa^{2} /(2 m-1)} u \mid m\right)$.

The peak value $\phi_{\max }$ of the variable $\phi$ (at $u=0$ ) is given by

$\phi_{\max }=\sqrt{\frac{m}{\left(1+\kappa^{2} / 4\right)(2 m-1)} \kappa^{2}}$ for $0<m \leq 1$.

The peak value of the corresponding angle variable $\theta$ (at $u=0$ ) is

$\theta_{\max }=\arctan \phi_{\max }$.

For $\phi_{\max }$ (and thus $\theta_{\max }$ ) to be real, positive values of $\kappa^{2}$ require $m>1 / 2$. Imaginary values of $\kappa$ are only possible in the range $-4<\kappa^{2}<0$, where the lower limit is set by the condition of having a positive compressive force $G_{0}$; in this case the requirement is $m<1 / 2$, with $\kappa=0$ for $m=1 / 2$. This defines the solution space that we will explore in Section 5, see Fig. 6.

As with other special functions, $c n(x \mid m)$ (and thus $\theta(u)$, Eq. (9)) is readily called up from, for example, Mathematica [44], as is the complete elliptic integral of the first kind $K(m)$, which gives its period. Examples were already shown in Figs. 3 and 5; several more will be shown in what follows.

For $m=1$ and $m=0$ the Jacobi functions simplify to more familiar forms, that is, $\operatorname{cn}(x \mid 1)=\operatorname{sech} x$ and $\operatorname{cn}(x \mid 0)=\cos x$, respectively. The corresponding scaled Jacobi functions $\phi(u)$ are listed in Table 1.

In the continuum representation of the single peak solutions, considered throughout this article, $N$ is half the period of the scaled Jacobi function, Eq. (9) (for the rescaled variable $u=$ $\left.x \sqrt{(2 m-1) / \kappa^{2}}\right)$, and thus given by

$N=2 K(m) \sqrt{\frac{2 m-1}{\kappa^{2}}}$,

where $K(m)$ is the complete elliptic integral of the first kind. For the case $N \rightarrow \infty\left(\kappa^{2} \rightarrow 0, m \neq 1 / 2\right)$ the solution asymptotes to zero in the limits $u \rightarrow \pm \infty$.

In the next section we shall examine the variety of these solutions for hard wall boundary conditions, an arbitrary number $N$ of spheres and an arbitrary value of compression $\Delta$, which are the parameters defining a typical experimental measurement.

\section{Solution diagrams}

The solution space is rich in detail, but the many parameters involved make it difficult to comprehend. The graphical method introduced here offers a transparent way to explore it.

Every solution for the specified boundary condition $(\phi, \theta=$ 0 at the end points, for $N$ spheres) must satisfy the reduced equation for some value of $\kappa^{2}$ (corresponding to the compressive force) and, from the above, can be expressed as an appropriately scaled Jacobi $c n$ function, Eq. (9), with squared modulus $m$. We will use the parameters, $m$ and $\kappa^{2}$, to label all of the solutions which they uniquely define when subjected to scaling. This leads us to construct "solution diagrams", with every solution (for the stated boundary conditions) represented by a point. We have found it convenient to use a nonlinear scale for $\kappa^{2}$ for graphical purposes, so that all values of $\kappa^{2}$ are captured on the diagrams. Hence, instead of $\kappa^{2}$, we use

$\hat{\kappa}^{2}=\kappa^{2} /\left(1+\left|\kappa^{2}\right|\right)$

when making these plots.

The form of such a diagram is shown in Fig. 6. This $\kappa^{2}-m$ plane will be our "playing field" in describing all of the different forms of solutions that can arise, and their variation.

The requirement of having a real value for $\theta_{\max }$, Eq. (11), leads to the identification of forbidden regions, i.e. areas in the $\kappa^{2}-m$ plane for which no solutions exist (see Section 4.2 ). The requirement $\kappa^{2}>-4$, i.e. $\hat{\kappa}^{2}>-\frac{4}{5}$ corresponds to a positive compressive force $G_{0}$. We will also, as discussed below, restrict ourselves to regions of the diagram for which the number of spheres, $N$, exceeds two. There is no such limitation inherent in the continuum formulation, but a contour defined by $N=2$ is to be regarded as representing the boundary beyond which the continuum model is unphysical.

Fig. 6 shows sample solutions for both negative and positive values of $\kappa^{2}$, together with the only solution at $\kappa^{2}=0$. Special forms (solution or absence of any finite solution) are to be found on the various boundaries of the allowed regions.

As anticipated in Section 3.2, the solutions are qualitatively different in the two allowed regions, as exemplified by those associated with the points $\mathrm{G}$ (for $\kappa^{2}<0$ ) and $\mathrm{C}$ (for $\kappa^{2}>0$ ). The first type has no inflection points, while the second has inflection points (at $\left.\theta=\arctan \sqrt{\kappa^{2} /\left(2\left(1+\kappa^{2} / 4\right)\right)}\right)$, giving rise to a localized peak.

In Fig. 7 we use our playing field to show how the number of spheres $N$ varies with $m$ and $\kappa^{2}$, by plotting contours of $N$ using Eq. (12). (The dependence of $N$ (periodicity) on the values for $\kappa^{2}$ and $m$ is also visible in the example solution of Fig. 6.) All of the contours pass through the point $m=1 / 2, \kappa^{2}=0$, so that the function $c n(x \mid m)$ can be scaled to provide a solution for $\kappa^{2}$ and any $N$. The contours asymptote to the $m$ axis as $N \rightarrow \infty$.

In Fig. 8 we show contours of constant maximum angle $\theta_{\max }$, Eq. (11). In an experiment, the number of spheres $N$ is constant, a change in compression (i.e. a change in $\kappa^{2}$ ) is thus represented by following a constant $N$ contour, see the examples for $N=5$ and $N=10$ shown in Fig. 8. $\theta_{\max }$ is seen to increase with $\kappa^{2}$ along such a contour. This is accompanied by an increase in the compressive strain, defined as $\epsilon=\Delta / N$, as can be seen in Fig. 9 which shows contours of constant $\epsilon$. 


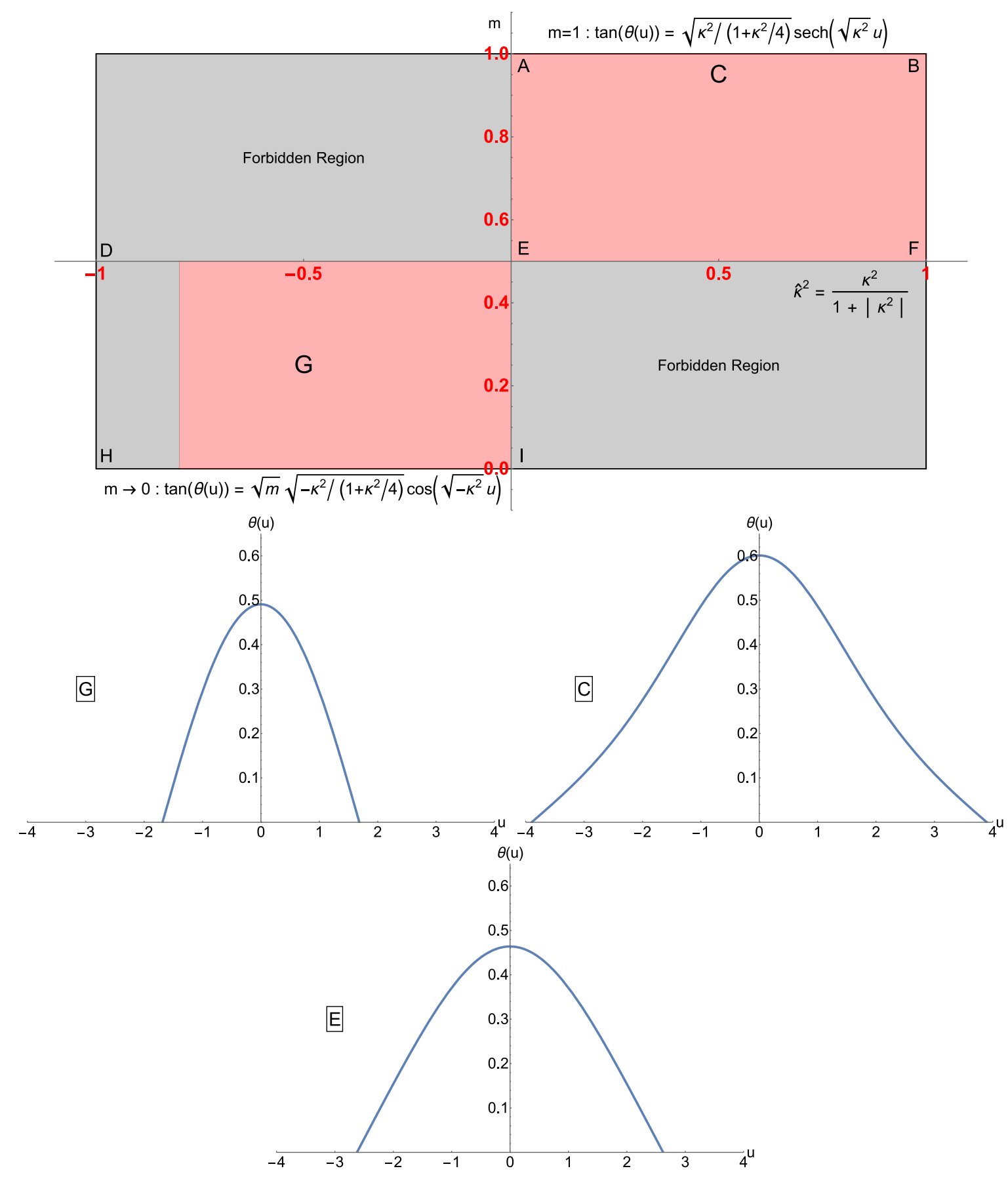

Fig. 6. Jacobi $c$ functions can be scaled to give solutions $\theta(u$ ) (Eq. (9)) of the reduced equation, Eq. (5), between two values of $u$ at which the boundary condition is $\theta=0$. These solutions are qualitatively different in the two pink domains indicated in the $\kappa^{2}-m$ "solution diagram", where $m^{1 / 2}$ is the modulus of the Jacobi function $\left(\hat{\kappa}\right.$ is defined by Eq. (13)). Inflection points only occur for $m>\frac{1}{2}$. Typical profiles are shown for the points $G,(-1 / 2,1 / 4)$, and $C,(1 / 2,0.95)$. Solutions at the boundary A-B have the following properties: $\theta_{\max }$ is finite and $N$ infinite, except at the limit; $\theta_{\max }$ is zero and $N$ is infinite at A. The intermediate solution at E, $(0,0.5)$, is given by the scaled Jacobi $c n$ function of roughly triangular form, $c n(\sqrt{2} u \mid 1 / 2)$. At $F,(1,1 / 2)$, the amplitude diverges while the period is zero.

An appropriate characteristic of a solution is its degree of localization. This may be defined by [45]

$D\left(m, \kappa^{2}\right)=\left(\int_{-N / 2}^{+N / 2} \theta(u) d u\right)^{2} /\left(N \int_{-N / 2}^{+N / 2} \theta^{2}(u) d u\right)$,

with $N$ given by Eq. (12),

This "participation ratio", used for example in the treatment of Anderson localization in solid state theory [46], answers the question: roughly speaking, over what proportion of the spheres is buckling concentrated?

Contours of constant participation ratio $D$ are shown in Fig. 10, where the integrals in Eq. (14) were computed numerically, using $\theta=\arctan \phi$ for our scaled Jacobi functions $\phi(u)$ (Eq. (9)). The smaller the value of $D$ the more localized is the solution, as in the limits of large $N$ and $\kappa^{2}$.

A further measure of localization which can be expressed analytically also for the case of the full differential equation, Eq. (2), is examined in Section 6. 


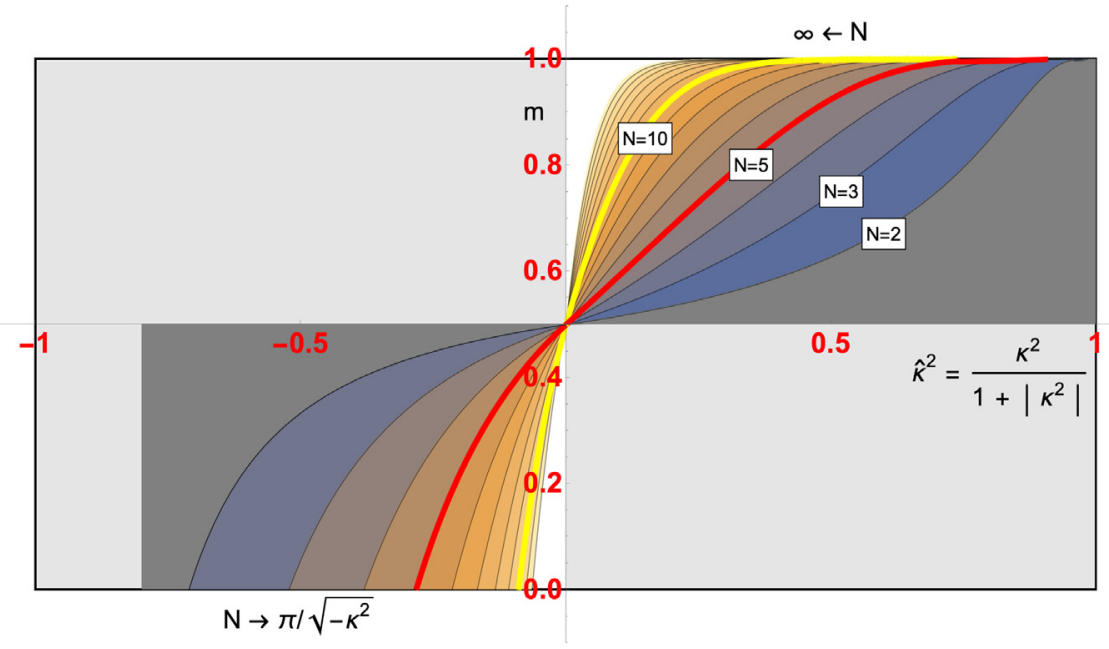

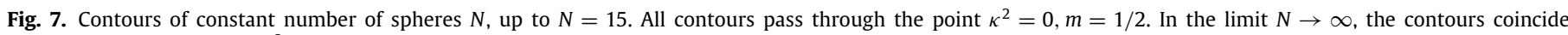

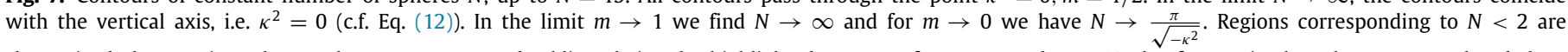

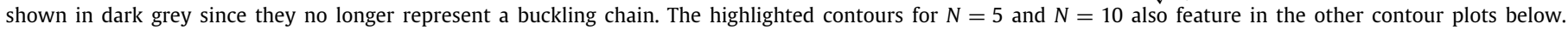
The case $N=10$ is the one shown in Figs. 2 and 3.

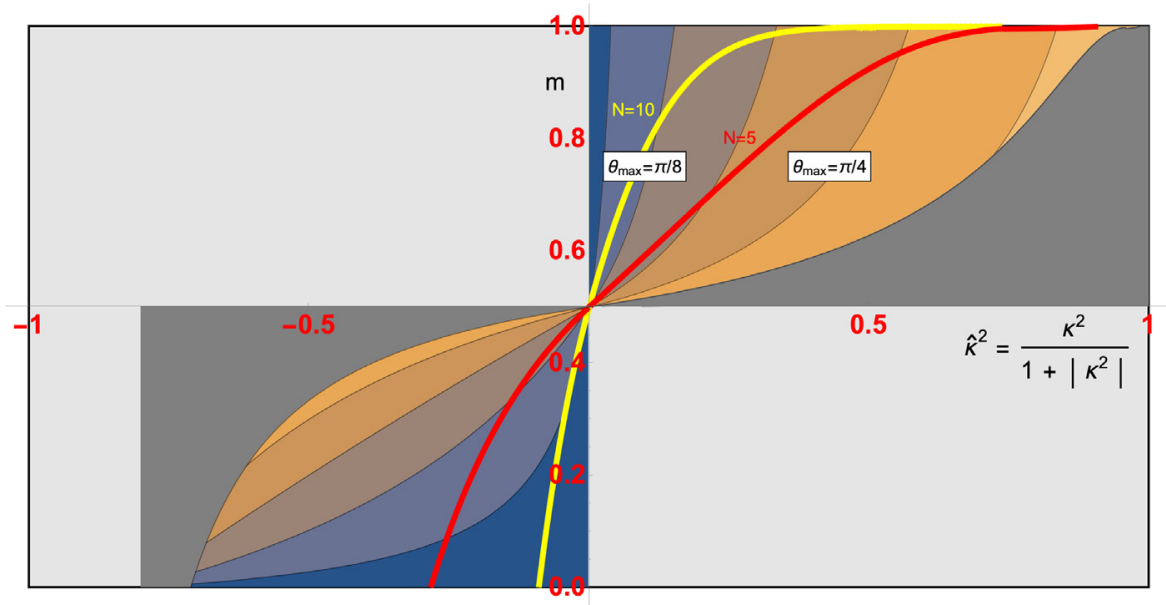

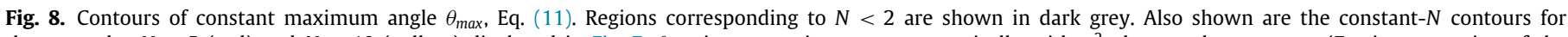

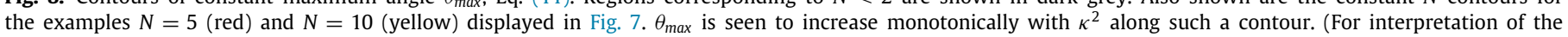
references to colour in this figure legend, the reader is referred to the web version of this article.)

\section{Estimate of accuracy of the reduced equation}

The previous sections described analytical solutions of the reduced equation, Eq. (5), in terms of scaled Jacobi functions. Here we will introduce a quantitative measure to assess how well the reduced equation approximates the full equation, Eq. (2). This involves the introduction of an alternative measure of localization, in terms of a peak width $w_{p}$, which can be expressed analytically for both equations.

The peak width $w_{p}$ may be defined as

$w_{p}=2 \sqrt{\frac{2 \theta_{\max }}{-\theta^{\prime \prime}\left(\theta_{\max }\right)}}$,

where $\theta_{\max }$ is the peak, and thus $\theta^{\prime \prime}\left(\theta_{\max }\right)<0$. (The quantity $w_{p}$ simply corresponds to the distance between the two zeros of an inverted parabola with maximum $\theta_{\max }$ at $u=0$.) The merit of this definition lies in providing an analytical expression for comparing solutions of the full equation to those of the reduced equation.
From the full equation, Eq. (2), we obtain

$w_{p, f u l l}=\sqrt{2 \theta_{\max }}\left[\sin \theta_{\max } \cos \theta_{\max }\left(1-\left(1+\frac{\kappa^{2}}{4}\right) \cos \theta_{\max }\right)\right]^{-1 / 2}$

while the reduced equation, Eq. (5), gives

$w_{p, \text { reduced }}=\sqrt{2 \theta_{\max }}\left[\sin \theta_{\max } \cos \theta_{\max }\left(\frac{1}{2}\left(1+\frac{\kappa^{2}}{4}\right) \tan ^{2} \theta_{\max }-\frac{\kappa^{2}}{4}\right)\right]^{-1 / 2}$

The relative difference in width $w_{p}$, defined as

$\epsilon_{w_{p}}=\left|w_{p, \text { full }}-w_{p, \text { reduced }}\right| / w_{p, \text { full }}$,

may then serve as an estimate of the error of the solutions of the reduced equation in relation to the full equation, Eq. (2); $\epsilon_{w_{p}}$ may be expressed analytically in terms of $\theta_{\max }$ and $\kappa^{2}$, using Eq. (16) and (17). 


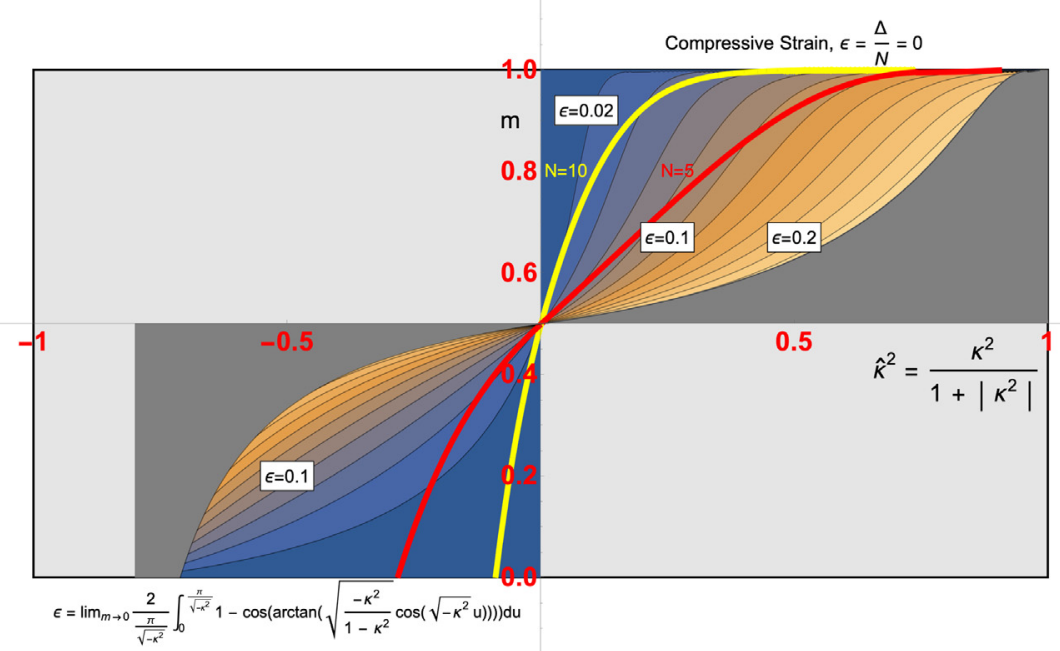

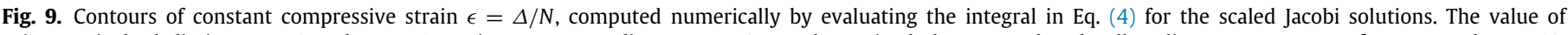

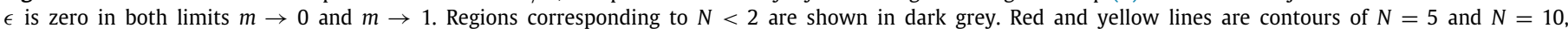

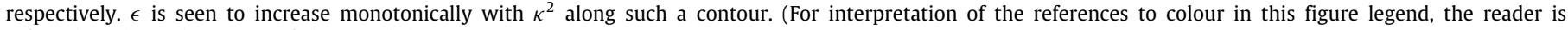
referred to the web version of this article.)

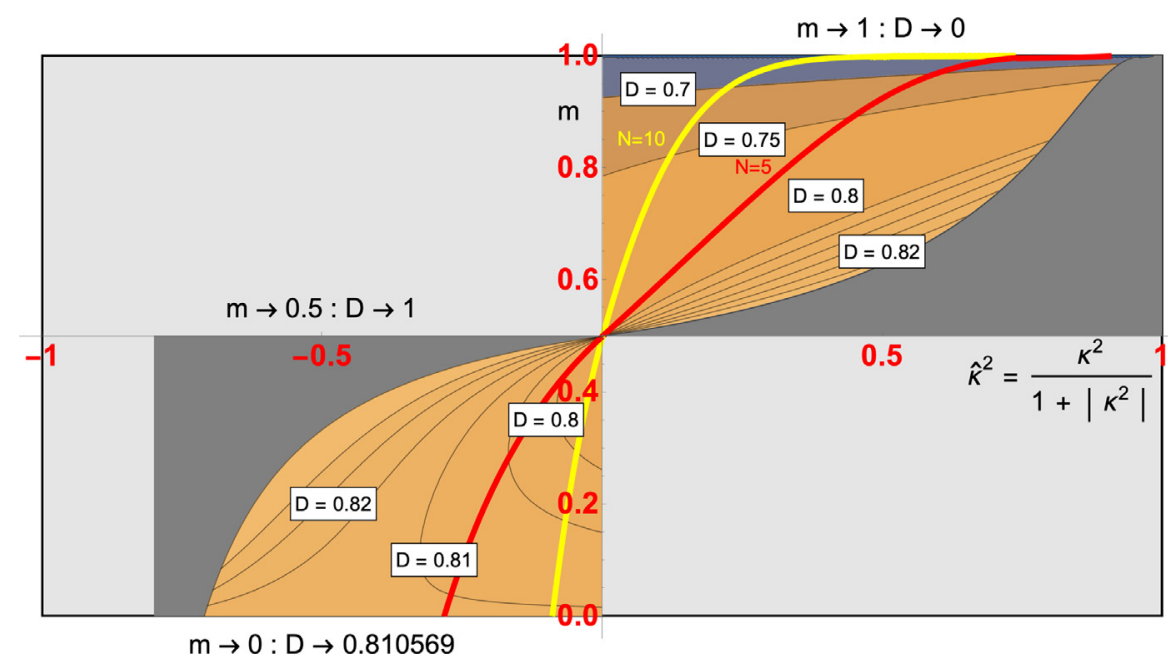

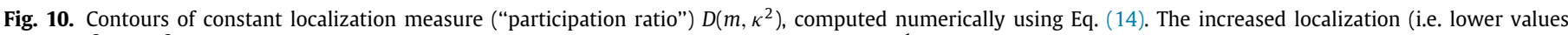

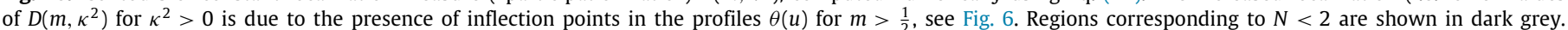

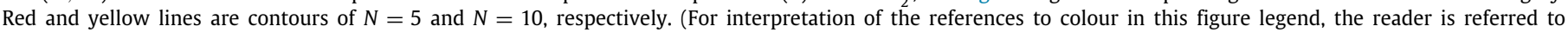
the web version of this article.)

Fig. 11 shows contours of constant error $\epsilon_{w_{p}}$. In line with our expectations the relative error vanishes in the limit of $\theta_{\max }, \kappa^{2} \rightarrow$ 0 , and thus of compression. The error is less than $10 \%$ even for values of $\theta_{\max } \simeq 0.5$, i.e. above the $\phi \ll 1$ limit of the reduced equation.

\section{Conclusion}

Localized buckling of the confined linear chain of spheres offers a problem for nonlinear theory which, inter alia, roughly represents localized failure in materials, and should repay further study, in particular by experiment $[3,4]$. Further generalization could incorporate, for example, elastic interactions. For such future work it will be convenient to have the background of analytic theory, as presented here.

Jacobi functions provide a full range of approximate solutions, easily evaluated and visualized, minimizing numerical calculations, elucidating qualitative features, and providing an accessible guide to the relationships of the several parameters $\left(N, \theta_{\max }, \Delta, D\right)$ that characterize the chain buckling.

It must be acknowledged that the continuum model fails to encompass one important aspect of the original discrete system: it does not produce the alternative asymmetric equilibrium profiles referred to earlier, which are not localized at the centre of the system [4]. These alternative equilibria are essential to an investigation of the Peierls-Nabarro potential, for example. We have undertaken a detailed study of this potential for the confined hard sphere system which again provides a straightforward context for its interpretation. It requires only the addition of a longitudinal force, modifying the equations presented here [47]. Other anticipated developments include changes of boundary conditions.

Looking further afield, we note that the confined linear chain of ions (with long-range Coulomb interactions) continues to attract interest, due to its possible application to quantum computing [15]. While numerical simulations of its buckling properties 


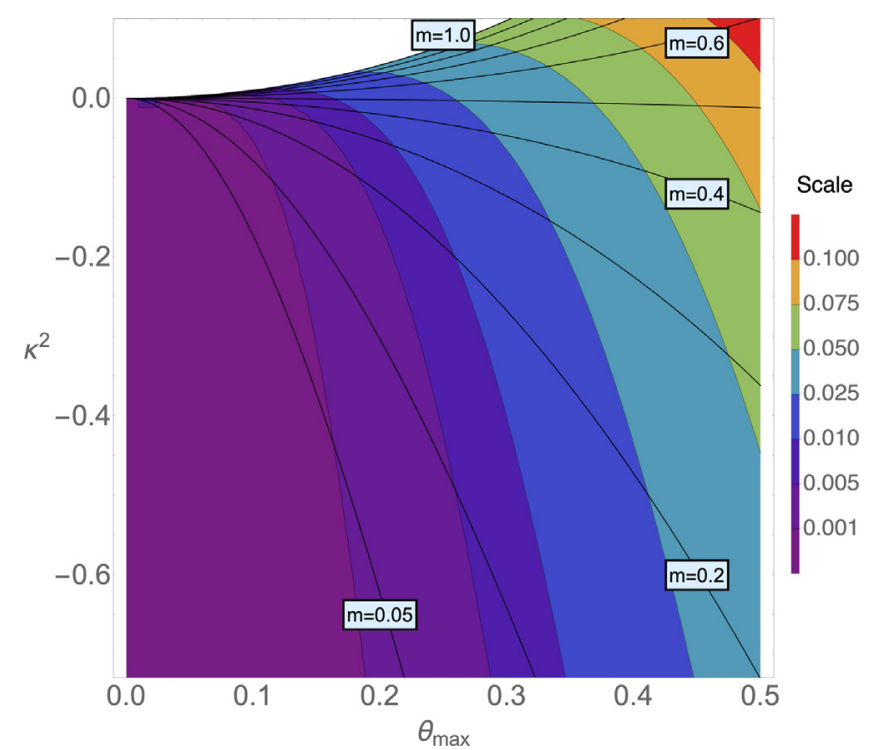

Fig. 11. Contour plot showing an estimate of the difference between solutions for the full equation and for the reduced equation. The contours are lines of constant values for $\epsilon_{w p}$, Eq. (18). Errors less than $10 \%$ are achieved for the shown range of $\kappa^{2}$ and $\theta_{\max }$. The black lines show the variation of $\kappa^{2}$ as a function of $\theta_{\max }$, computed from Eq. (11), for six different values of the squared Jacobi modulus $m$. Only values of $\left(\theta_{\max }, \kappa^{2}\right)$ lying below the line for $m=1$ are possible.

have been investigated to some extent, including the computation of a Peierls-Nabarro potential [9], results and interpretations remain limited. The mathematical description of this system is significantly more difficult, however, we expect that some aspects of our analysis will prove fruitful for its understanding.

Another practical problem in which buckling plays a role is the design of 'mechanical meta-materials'. A key feature of such systems is that the mechanical properties of the system emerge as a consequence of geometry as opposed to the material composition of the system [48]. Examples of such systems include linear columns with regularly arranged voids; under compression such porous columns are found to buckle, causing the voids to deform in a variety of modes [49,50]. The present work, while for a series of hard spheres, displays many of the same nonlinear behaviours as observed for the porous columns. As such, some of the analysis developed here may find applications in the study of various meta-material designs.

The confined and compressed chain model developed here was invoked in order to describe particular experimental systems, as explained in Section 1. It has much wider relevance, as a transparent illustration of the general phenomena of buckling, bifurcation etc.

\section{CRediT authorship contribution statement}

D. Weaire: Original draft, Review \& editing. A. Mughal: Software, Visualization, Investigation. J. Ryan-Purcell: Investigation, Software. S. Hutzler: Supervision, Validation, Review \& editing.

\section{Declaration of competing interest}

The authors declare that they have no known competing financial interests or personal relationships that could have appeared to influence the work reported in this paper.

\section{Acknowledgements}

SH was supported by Science Foundation Ireland (SFI) grant 13/IA/1926 and the European Space Agency SciSpacE program (project "REFOAM", contract number 4000129502/20/NL/PG). AM acknowledges the support of the Supercomputing Wales project, which is part-funded by the European Regional Development Fund (ERDF) via Welsh Government. We thank one of the reviewers of an earlier version of this manuscript for the very helpful suggestion regarding the reduction of the differential equation (Appendix C), resulting in an improvement over our previous version presented in [4].

\section{Appendix A. Modelling the discrete system}

A chain of $N$ contacting identical hard spheres is compressed between hard walls, as in Fig. A.1. Displacement of a sphere by a distance $R_{n}$ away from the central axis results in a transverse restoring force $f_{n}$ with magnitude $k R_{n}$, where $k$ is a force constant. We introduce non-dimensional quantities by defining $r_{n}=R_{n} / D$, where $D$ is the sphere diameter. The dimensionless transverse force $F_{n}$ is defined as $F_{n}=f_{n} /(k D)$. Inserting for $f_{n}$ leads to $F_{n}=r_{n}$; in our non-dimensional formulation the transverse force $F_{n}$ acting on a sphere equals its transverse displacement $r_{n}$, a positive quantity. by

The compressive forces between contacting spheres are given

$G_{n} \cos \theta_{n-1}=G_{0}$

from the condition of force equilibrium, where $G_{0}$ is the magnitude of the compressive force at each end of the system. As with $F_{n}$ we will express the compressive forces $G_{n}$ and $G_{0}$ as non-dimensional quantities (division by $k D$ ) [2].

Transverse force balance for the displaced $n^{\text {th }}$ sphere is expressed as

$F_{n}=G_{n} \sin \theta_{n-1}+G_{n+1} \sin \theta_{n}=G_{0}\left(\tan \theta_{n-1}+\tan \theta_{n}\right)$.

The centres of contacting spheres are separated by their diameter. Hence in our dimensionless variables the radial distances and forces are given by

$F_{n}+F_{n+1}=r_{n}+r_{n+1}=\sin \theta_{n}$.

Eq. (A.2) and (A.3) provide an iterative scheme for relating $\theta_{n+1}$ and $F_{n+1}$ to $\theta_{n}$ and $F_{n}$.

$$
\begin{array}{r}
\theta_{n}=\arctan \left(\frac{F_{n}}{G_{0}}-\tan \theta_{n-1}\right) \\
F_{n+1}=\sin \theta_{n}-F_{n}
\end{array}
$$

These equations can be used in a shooting method to find solutions for a specified value for $G_{0}[2,4]$. The hard wall boundary condition for sphere $n=1$ requires the first angle $\theta_{0}$ to be zero, with an arbitrary $F_{1}$. The spheres are not confined to the axis, $F_{1}$ and $F_{N}$ (and thus $r_{1}$ and $r_{N}$ ) can differ from zero.

Using Eq. (A.4) we proceed iteratively to $\left(\theta_{N}, F_{N}\right)$. The angle $\theta_{N}$ corresponds to the contact of the $N^{\text {th }}$ sphere with the wall, which can be made equal to zero by adjusting the value of $F_{1}[2,4]$.

Compression $\Delta$ is defined as $\Delta=(N D-L) / D$, where $L$ is the total chain length. This results in

$$
\Delta=N-\sum_{n=0}^{N} \cos \theta_{n} \text {. }
$$

\section{Appendix B. The uniform zig-zag structure}

If periodic boundary conditions are applied to a finite chain (with even sphere number) then a simple solution is provided by a sphere arrangement where all transverse displacements are equal (and alternate in sign). This corresponds to constant values 


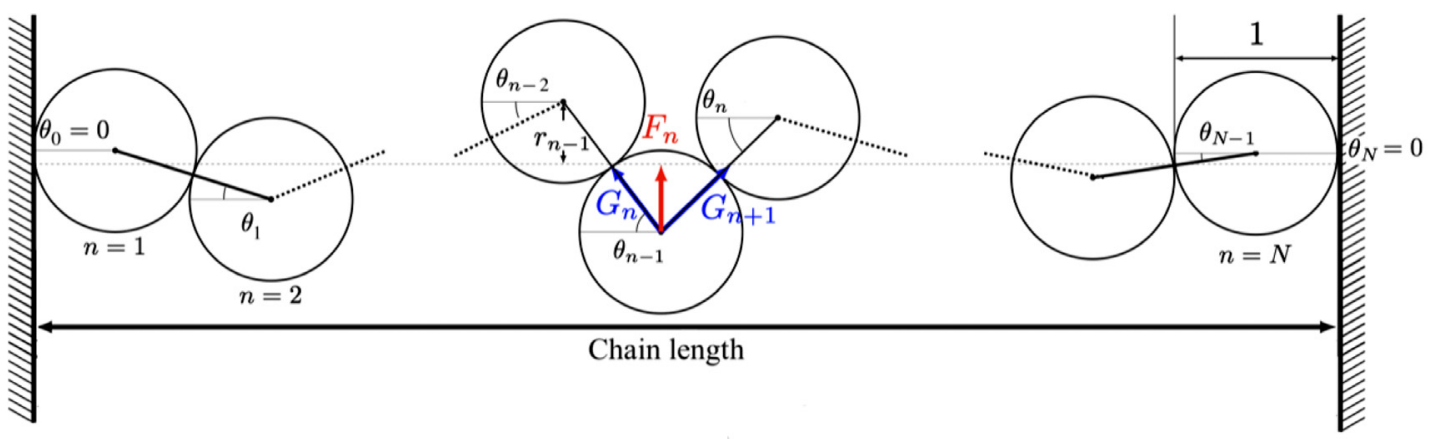

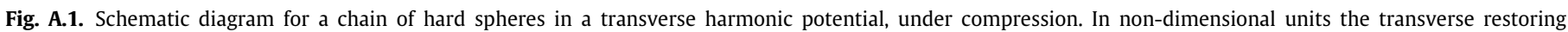

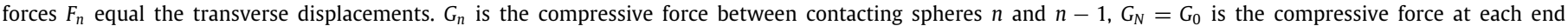

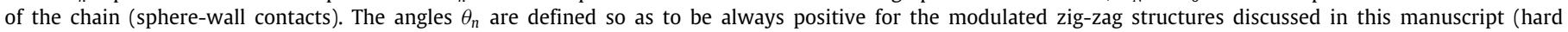
walls: $\theta_{0}=\theta_{N}=0$ ).

for $\theta_{n}, F_{n}$ and $G_{n}$ for all $n$. We refer to this as the uniform zigzag structure [4], and all solutions discussed in this paper are modulations of it.

From Eq. (A.2) we obtain the following relationship between transverse forces (displacements) $F$ and compressive forces $G$, for this structure

$F=2 G \sin \theta$.

The geometry of the zig-zag structure (see also Eq. (A.3)) results in $\sin \theta=2 F$, i.e. $G=1 / 4$ for the compressive force between contacting spheres, independent of the value of compression. The longitudinal component of this force, $G_{0}$ is thus given by

$G_{0}=\frac{1}{4} \cos \theta$.

(We note that in $[2,4]$ we erroneously wrote $G_{0}=1 / 4$, i.e. the limiting value for small transverse displacements.) The compressive force at each end of the chain thus decreases with compression, since compression leads to an increase in the angle $\theta$. This implies the instability of the uniform zig-zag structure.

\section{Appendix C. Transition to the continuum formulation}

In a continuum description both the angle $\theta$ and the transverse force $F$ are functions of a continuous variable $u$. Compression $\Delta$, Eq. (A.5), is then obtained from evaluation of the following integral,

$\Delta=\int_{0}^{N}(1-\cos \theta(u)) d u$.

The approximate continuum representation of the iterative relations Eq. (A.4) may be obtained as follows. By introducing a new variable $\phi_{n}$ as $\phi_{n}=\tan \theta_{n}$, we re-express the two iterative Eqs. (A.4) as

$$
\begin{array}{r}
\phi_{n}=\frac{F_{n}}{G_{0}}-\phi_{n-1} \\
F_{n+1}=\frac{\phi_{n}}{\sqrt{1+\phi_{n}^{2}}}-F_{n},
\end{array}
$$

where we used the identity $\sin \theta_{n}=\tan \theta_{n} / \sqrt{1+\tan ^{2} \theta_{n}}$.

Using the first of these equations we obtain

$F_{n}+F_{n+1}=G_{0}\left(\phi_{n+1}+2 \phi_{n}+\phi_{n-1}\right)$.

Re-expressing the left hand side using the second iterative equation in Eq. (C.2) results in

$\frac{\phi_{n}}{\sqrt{1+\phi_{n}^{2}}}=G_{0}\left(\phi_{n+1}-2 \phi_{n}+\phi_{n-1}\right)+4 G_{0} \phi_{n}$.
The term in brackets on the r.h.s. may be identified as a central difference approximation of the second derivative of a continuous function $\phi(u)$ with respect to a continuous variable $u$, evaluated at $u=n$. A continuum formulation of this equation is thus given by

$\phi^{\prime \prime}(u)=-4 \phi(u)+\frac{\phi(u)}{G_{0} \sqrt{1+\phi^{2}(u)}}$.

$G_{0}$ is the compressive force exerted at both ends of the chain (see Appendix A). We find it convenient to express it in terms of a complex number $\kappa$, so that $\kappa^{2}$ may take on positive or negative values,

$G_{0}=\left(4+\kappa^{2}\right)^{-1}$.

This choice of the definition is motivated by the value $G_{0} \simeq 1 / 4$ for the uniform zig-zag structure discussed in Appendix B.

The second order differential equation for the continuum formulation for our buckling chain is thus given by

$\phi^{\prime \prime}(u)=-4 \phi(u)+\left(4+\kappa^{2}\right) \frac{\phi(u)}{\sqrt{1+\phi^{2}(u)}}$.

Eq. (C.7) can be solved numerically, using for example Mathematica. However, we found it useful to expand the square root to order $\phi^{2}$. This results in

$\phi^{\prime \prime}=\kappa^{2} \phi-2\left(1+\frac{\kappa^{2}}{4}\right) \phi^{3}$,

correct to order $\mathcal{O}\left(\phi^{3}\right)$. We call Eq. (C.8) the reduced equation. Having a finite positive compressive force $G_{0}$ at both ends of the chain of spheres requires $\kappa^{2}>-4$ (from Eq. (C.6)).

In previous work [4] we presented a reduced equation which corresponds to approximating $\phi=\tan \theta$ by $\theta$ here and neglecting the $\kappa^{2}$ term in the bracket. Clearly this has some validity for small $\theta(\tan \theta \simeq \theta)$. Alternative derivations of the continuum equation will be explored in future work [51].

\section{Appendix D. Rescaling of Jacobi functions}

We will show that a solution $\phi(u)$ which satisfies the reduced equation, Eq. (5), (C.8), can be expressed in terms of a Jacobi function $y(x)=c n(x \mid m)$ for appropriately rescaled variables. Back-substitution then results in the angle profile $\theta(u)=$ $\arctan \phi(u)$.

We proceed by introducing real constants $c_{1}$ and $c_{2}$, whose value remains to be determined, and set $\phi=c_{1} y$ and $u=c_{2} x$. The second derivative of $\phi$ with respect to $u$ is thus given by $\frac{d^{2} \phi}{d u^{2}}=\frac{c_{1}}{c_{2}^{2}} \frac{d^{2} y}{d x^{2}}$. 
In the new variables $y(x)$ the reduced equation, Eq. (5), is rewritten as $\frac{c_{1}}{c_{2}^{2}} \frac{d^{2} y}{d x^{2}}=\kappa^{2} c_{1} y-2 c_{1}^{3} y^{3}$. This results in our key equation for further analysis,

$\frac{d^{2} y}{d x^{2}}=\kappa^{2} c_{2}^{2} y-2 c_{1}^{2} c_{2}^{2} y^{3}$.

In this rescaled equation the $y^{3}$ term features with a minus sign. This rules out 8 of the 12 Jacobi functions.

Eq. (D.1) is solved by the Jacobi $c n(x \mid m)$ function if we set $c_{1}^{2} c_{2}^{2}=m$ and $\kappa^{2} c_{2}^{2}=2 m-1$. Solving for $c_{1}^{2}$ and $c_{2}^{2}$ we obtain $c_{2}^{2}=\frac{2 m-1}{\kappa^{2}}$ and $c_{1}^{2}=\frac{m \kappa^{2}}{\left(1+\kappa^{2} / 4\right)(2 m-1)}$.

Using $\phi=c_{1} y$ and $x \stackrel{u}{=} u / c_{2}$ from above yields our scaled Jacobi cn solution $\phi(u)$ of the reduced Eq. (5),

$\phi(u)=\tan \theta(u)=\sqrt{\frac{m \kappa^{2}}{\left(1+\kappa^{2} / 4\right)(2 m-1)}} c n\left(\sqrt{\frac{\kappa^{2}}{2 m-1}} u \mid m\right)$,

with $\kappa^{2}>-4$, as discussed in Appendix C.

We have $0 \leq m \leq 1$, this requires $\kappa^{2}<0$ for $m<1 / 2$ and $\kappa^{2}>0$ for $m>1 / 2 . m=1 / 2$ can only lead to a solution for $\kappa^{2}=0$ (division $\left.0 / 0\right)$.

Limiting cases:

$m \rightarrow 0,-4<\kappa^{2}<0: \tan \theta(u) \rightarrow \sqrt{m} \sqrt{-\kappa^{2} /\left(1+\kappa^{2} / 4\right)}$ $\cos \left(\sqrt{-\kappa^{2}} u\right)$

$m=\frac{1}{2}, \kappa^{2}=0: \tan \theta(u)=c n\left(\sqrt{2} u \mid \frac{1}{2}\right)$

$m=1, \kappa^{2}>0: \tan \theta(u)=\sqrt{\kappa^{2} /\left(1+\kappa^{2} / 4\right)} \operatorname{sech}\left(\sqrt{\kappa^{2}} u\right)$

Here the case for $m=\frac{1}{2}$ can be obtained by setting $\sqrt{\frac{m \kappa^{2}}{\left(1+\kappa^{2} / 4\right)(2 m-1)}}=1$ and $\frac{\kappa^{2}}{2 m-1}=2$, from which it follows that $m=\frac{1}{2}$ and $\kappa^{2}=0$.

\section{Appendix E. Relevance to the pendulum}

The reduced equation, Eq. (5), is also relevant to the theory of the mathematical pendulum. The pendulum equation for the angle of displacement $y(t)$ from the vertical is

$\ddot{y}(t)=-\omega_{0}^{2} \sin y(t)$,

where $\omega_{0}^{2}$ is the angular frequency for small amplitude oscillations (i.e. the case of the harmonic oscillator). An exact solution for all amplitudes may be expressed in terms of Jacobi functions [41,52], but various transformations are required to derive this. An intermediate approximation can be applied using

$\sin y \simeq y-y^{3} / 6$,

resulting in

$\ddot{y}(t)=-\omega_{0}^{2} y(t)+\frac{\omega_{0}^{2}}{6} y^{3}(t)$.

This equation may be treated in the manner of Section 4.2 to derive a solution in terms of the Jacobi $c d$ function,

$y(t)=y_{0} c d\left(\omega_{0} \sqrt{1-\frac{y_{0}^{2}}{12}} t \mid \frac{y_{0}^{2}}{12-y_{0}^{2}}\right)$,

with initial displacement $y_{0}=y(t=0)$.

The period of this solution, $T=\frac{2 \pi}{\omega_{0}}\left(1+\frac{y_{0}^{2}}{16}+\mathcal{O}\left(y_{0}^{4}\right)\right)$ agrees with that of the mathematical pendulum up to order $y_{0}^{2}$.

\section{References}

[1] T. Lee, K. Gizynski, B.A. Grzybowski, Adv. Mater. 29 (2017) 1704274.

[2] J. Winkelmann, A. Mughal, D. Weaire, S. Hutzler, Europhys. Lett. 127 (2019) 44002.

[3] D. Weaire, A. Irannezhad, A. Mughal, S. Hutzler, Amer. J. Phys. 88 (2020) 347.

[4] S. Hutzler, A. Mughal, J. Ryan-Purcell, A. Irannezhad, D. Weaire, Phys. Rev. E 102 (2020) 022905.

[5] S. Ejtemaee, P.C. Haljan, Phys. Rev. A 87 (2013) 051401.

[6] M. Mielenz, J. Brox, S. Kahra, G. Leschhorn, M. Albert, T. Schätz, H. Landa, B. Reznik, Phys. Rev. Lett. 110 (2013) 133004.

[7] J. Pyka, H. Keller, R. Partner, T. Nigmatullin, D. Burgermeister, K. Meier, A. Kuhlmann, M.B. Retzker, K. Plenio, W. Zurek, et al., Nature Commun. 4 (2013) 1 .

[8] H. Landa, B. Reznik, J. Brox, M. Mielenz, T. Schätz, New J. Phys. 15 (2013) 093003.

[9] H.L. Partner, R. Nigmatullin, T. Burgermeister, K. Pyka, J. Keller, A. Retzker, M.B. Plenio, T.E. Mehlstäubler, New J. Phys. 15 (2013) 103013.

[10] R.C. Thompson, Contemp. Phys. 56 (2015) 63.

[11] H.L. Partner, R. Nigmatullin, T. Burgermeister, J. Keller, K. Pyka, M.B. Plenio, A. Retzker, W.H. Zurek, A. del Campo, T.E. Mehlstäubler, Physica B 460 (2015) 114

[12] R. Nigmatullin, A. del Campo, G. De Chiara, G. Morigi, M.B. Plenio, A. Retzker, Phys. Rev. B 93 (2016) 014106.

[13] L. Yan, W. Wan, L. Chen, F. Zhou, S. Gong, X. Tong, M. Feng, Sci. Rep. 6 (2016) 21547.

[14] H. Landa, S. Marcovitch, A. Retzker, M.B. Plenio, B. Reznik, Phys. Rev. Lett. 104 (2010) 043004.

[15] I. Georgescu, Nat. Rev. Phys. 2 (2020) 278

[16] A. Melzer, Phys. Rev. E 73 (2006) 056404.

[17] T. Beatus, T. Tlusty, R. Bar-Ziv, Nat. Phys. 2 (2006) 743.

[18] A.V. Straube, R.P. Dullens, L. Schimansky-Geier, A.A. Louis, J. Chem. Phys. 139 (2013) 134908.

[19] L. Helseth, T. Johansen, T. Fischer, Phys. Rev. E 71 (2005) 062402.

[20] https://physicsworld.com/a/physics-in-the-pandemic-there-are-somegreat-experiments-that-can-be-done-safely-and-simply-at-home/.

[21] O.M. Braun, Y.S. Kivshar, Phys. Rep. 306 (1998) 1.

[22] F.F. Dunne, J. Winkelmann, D. Weaire, S. Hutzler, Phil. Mag. 99 (2019) 2303-2320.

[23] J.E. Gordon, The new science of strong materials, in: Pelican Books, second ed., 1976

[24] L.P. Lebedev, M.J. Cloud, Approximating Perfection, Princeton University Press, 2004.

[25] L.J. Gibson, M.F. Ashby, Cellular Materials, Cambridge University Press, 1997.

[26] T.C.T. Michaels, R. Kusters, A.J. Dear, C. Storm, J.C. Weaver, L. Mahadevan, Proc. Math. Phys. Eng. Sci. 4 (2019) 249

[27] J.Y. Chung, A. Vaziri, L. Mahadevan, Proc. Natl. Acad. Sci. USA 115 (2019) 7509.

[28] R.M.J. Groh, G.W. Hunt, A. Pirrera, Int. J. Mech. Sci. 196 (2021) 106297.

[29] D.E. Wolf, Modelling and computer simulation of granular media, in: K.H. Hoffmann, M. Schreiber (Eds.), Computational Physics, Springer Verlag, 1996, pp. 64-95.

[30] A. Tordesillas, Stranger than friction: force chain buckling and its implications for constitutive modelling, in: T. Aste, T. Di Matteo, A. Tordesillas (Eds.), Granular and Complex Materials, World Scientific, 2007, pp. 95-109.

[31] M.C. Jenkins, M.D. Haw, G.C. Barker, W.C. Poon, S.U. Egelhaaf, Soft Matter 7 (2011) 684-690.

[32] D.C. Morse, T.A. Witten, Europhys. Lett. 22 (1993) 549-555.

[33] R. Höhler, D. Weaire, Adv. Colloid Interface Sci. 263 (2019) 19-37.

[34] G.W. Hunt, A. Tordesillas, S.C. Green, J. Shi, Phil. Trans. R. Soc. A 368 (2010) 249.

[35] I. Kovacic, M.J. Brennan, The Duffing Equation: Nonlinear Oscillators and their Behaviour, John Wiley \& Sons, 2011.

[36] A.J. Brizard, Eur. J. Phys. 30 (2009) 729.

[37] K.R. Meyer, Amer. Math. Monthly 108 (2001) 729.

[38] W.A. Schwalm, Lectures on Selected Topics in Mathematical Physics: Elliptic Functions and Elliptic Integrals, Morgan \& Claypool Publishers, 2015.

[39] Y.V. Zakharov, K. Okhotkin, J. Appl. Mech. Tech. Phys. 43 (2002) 739.

[40] A. Beléndez, C. Pascual, D.I. Méndez, T. Beléndez, C. Neipp, Rev. Brasileira Ensino FíSica 29 (2007) 645-648.

[41] D.F. Lawden, Elliptic Functions and Applications, Vol. 80, Springer Science \& Business Media, 2013.

[42] P.F. Byrd, M.D. Friedman, Handbook of Elliptic Integrals for Engineers and Physicists, Vol. 67, Springer, 2013. 
[43] M. Abramowitz, Appl. Math. Ser. (1964).

[44] Mathematica, Version 12.1, Wolfram Research Inc.

[45] D. Weaire, A. Williams, J. Phys. C Solid State Phys. 9 (1976) L461.

[46] V. Srivastava, D. Weaire, Phys. Rev. B 18 (1978) 6635.

[47] A. Mughal, D. Weaire, S. Hutzler, Europhys. Lett. 135 (2021) 26002.
[48] X. Yu, J. Zhou, H. Liang, Z. Jiang, L. Wu, Prog. Mater. Sci. 94 (2018) 114

[49] G. Oliveri, J.T.B. Overvelde, Adv. Funct. Mater. 30 (2020) 1909033.

[50] A. Nazir, A.B. Arshad, J.-Y. Jeng, J. Jeng-Ywan, Materials 12 (2019) 3539.

[51] D. Weaire, A. Mughal, J. Ryan-Purcell, S. Hutzler, in preparation (2022).

[52] E.T. Whittaker, A treatise on the analytical dynamics of particles and rigid bodies, 1937, CUP Archive. 